\title{
A randomised controlled trial of domiciliary and hospital-based rehabilitation for stroke patients after discharge from hospital
}

\author{
J R F Gladman, N B Lincoln, D H Barer
}

\begin{abstract}
This study compared the functional ability and perceived health status of stroke patients treated by a domiciliary rehabilitation team or by routine hospital-based services after discharge from hospital. Patients discharged from two acute and three rehabilitation hospitals in Nottingham were randomly allocated in three strata (Health Care of the Elderly, General Medical and Stroke Unit) to receive domiciliary or hospital-based care after discharge. Functional recovery was assessed by the Extended Activities of Daily Living (ADL) scale three and six months after discharge and perceived health at six months was measured by the Nottingham Health Profile. A total of 327 eligible patients of 1119 on a register of acute stroke admissions were recruited over 16 months. Overall there were no differences between the groups in their Extended ADL scores at three or six months, or their Nottingham Health Profile scores at six months. In the Stroke Unit stratum, patients treated by the domiciliary team had higher household $(p=0.02)$ and leisure activity $(p=$ $0.04)$ scores at six months than those receiving routine care. In the Health Care of the Elderly stratum, death or a move into long-term institutional care at six months occurred less frequently in patients allocated to the routine service, about half of whom attended a geriatric day hospital. Overall there was no difference in the effectiveness of the domiciliary and hospital-based services, although younger stroke unit patients appeared to do better with home therapy while some frail elderly patients might have benefited from day hospital attendance.
\end{abstract}

$(\Im$ Neurol Neurosurg Psychiatry 1993;56:960-966)

The advantages of early intervention by a well-organised rehabilitation service in speeding the recovery of patients admitted to hospital with a stroke have been demonstrated in controlled trials, ${ }^{12}$ but a high proportion of patients deteriorate after discharge so that early gains may be lost. ${ }^{3}$ Outpatient rehabilitation aims to prevent such deterioration and promote the re-acquisition of activities of daily living (ADL) such as shopping, housework and leisure activities ("instrumental"
$\mathrm{ADL}$ ), to return patients as close as possible to their previous way of life.

A study at Northwick Park ${ }^{4}$ comparing different intensities of outpatient hospitalbased rehabilitation suggested that stroke patients receiving the maximum treatment (four full days a week) did best, but the advantage was small and the main benefit appeared to be in preventing deterioration. Only $18 \%$ of patients discharged from hospital were thought suitable for the treatment, one in three patients being judged too old or frail to make frequent visits to hospital. ${ }^{5}$ There is no reason to suppose that frailer people would not have benefited from therapy if it could have been provided at home. Indeed, there are many potential advantages of domiciliary rehabilitation. The patient is seen in his/her own surroundings, where it may be easier to identify real practical problems and to practise the solutions to them than in the alien environment of a hospital. The family can be closely involved in the rehabilitation process and the difficulties and worries of carers ${ }^{6}$ can be directly addressed. The erratic provision of services and aids ${ }^{7}$ can be monitored and a coordinated rehabilitation programme developed in conjunction with community services. Finally, the stresses of ambulance journeys to hospital ${ }^{8}$ can be avoided. On the other hand, the centralisation of rehabilitation services in outpatient departments or day hospitals offers potential advantages in terms of efficient use of resources.

A trial of a home care service for stroke patients in Bristol ${ }^{9}$ showed no specific advantage over conventional hospital-based care, but the small team spent much of its efforts on providing support for patients in the acute stages of stroke and trying to prevent admission to hospital. Thus neither the resources available nor the study design may have been adequate to test the effectiveness of domiciliary rehabilitation. The results of a recent controlled trial in Bradford $^{10}$ indicate that physiotherapy for stroke patients at home is more effective in reducing disability than rehabilitation at a day hospital.

While the study in Bradford was in progress we conducted a randomised controlled trial of a domiciliary rehabilitation team including physiotherapy and occupational therapy to determine whether stroke patients would make greater improvements in instrumental $\mathrm{ADL}$ after discharge from hospital and whether carer stress could be reduced in comparison with patients treated by the conventional hospital-based rehabilitation service. 


\section{Methods}

Patients were identified from a register of all those admitted to the City and University Hospitals, Nottingham with acute stroke. All patients on the register (including those with recurrent strokes) met World Health Organisation clinical criteria for acute stroke ${ }^{11}$ and were seen by a single observer (JG). Demographic and clinical characteristics were recorded. Patients were considered for the study when plans for discharge were being made. Those discharged to residential or nursing homes were excluded, as were those requiring respite or terminal care, those who had been receiving outpatient rehabilitation before the stroke, those who had no significant disability from their stroke and those who stayed in hospital for less than 7 days.

Once patients were considered suitable for the study, informed consent was obtained. A history of vascular disease, urinary incontinence, mental disability or immobility (requiring help from another person to walk) was recorded as well as the current Barthel ADL score, ${ }^{1213}$ Abbreviated Mental Test Score ${ }^{14}$ and the presence or absence of aphasia. Patients were then randomly allocated to either the domiciliary or hospital-based rehabilitation service. This was done by opening consecutive sealed envelopes which contained cards marked either "DRS" or "HRS", which had been prepared before the start of the study by reference to a table of random numbers. Separate randomisation schedules were provided for patients discharged from the Health Care of the Elderly wards, General Medical wards or the Stroke Unit, because our pilot work ${ }^{15}$ had shown that the characteristics of these groups of patients and the usual rehabilitation services provided for them after discharge from hospital differed considerably. Ethical approval for the study was obtained.

The domiciliary service was provided by 2 half time physiotherapists and 1 occupational therapist who assessed all patients referred to it at home then organised or provided appropriate therapy and arranged other relevant help. Patients were treated by the domiciliary team for up to six months, after which those requiring further rehabilitation were referred back to the routine services. Since the domiciliary service was new, a run-in period of four months was allowed before the randomised trial began and the members of the domiciliary team visited domiciliary services in other areas. The aim was to operate a service along the usual lines of a NHS service.

Patients allocated to the hospital-based rehabilitation service were eligible for outpatient rehabilitation according to the usual practices in Nottingham, where there had hitherto been no domiciliary rehabilitation service. For patients discharged from Health Care of the Elderly wards the main option was a day hospital while for patients on General Medical wards, outpatient physiotherapy or occupational therapy could be arranged. A few Health Care of the Elderly patients were referred to outpatient therapy departments and a minority of General Medical patients were referred to a geriatrician for day hospital follow up.

The primary outcome measure, assessed three and six months after randomisation, was the overall Extended ADL score, ${ }^{16}$ a postal assessment of instrumental ADL with separate sections for mobility, household and leisure activities. ${ }^{17}$ At six months an independent observer, who was unaware of the treatment group, assessed personal ADL using the Barthel Index and perceived health using the Nottingham Health Profile, ${ }^{18}$ administered at interview. Informal carers (living with the patient) were also assessed at six months using the Brief Assessment of Social Engagement ${ }^{1920}$ and the Nottingham version of the Life Satisfaction Index. ${ }^{20} 21$

Data were analysed on the University of Nottingham mainframe computer using the Statistical Package for the Social Sciences and MINITAB. Non-parametric significance tests (Chi square and Mann-Whitney) were used because the level of measurement of $A D L$ scales is at best ordinal.

The calculation of sample size was based on a standard deviation of 6 points in the Extended ADL scale observed during the run-in period. Using a parametric model ${ }^{22}$ the sample size needed to detect a mean difference of 2 Extended $\mathrm{ADL}$ points, at the 5\% significance level, with a power of $80 \%$ was 150 patients per group. To allow for dropouts and for the reduced power efficiency of distribution-free tests the total study size was set at about 320 patients.

\section{Results}

PATIENTS

Between the beginning of April 1989 and the end of July 1990, 1119 patients with acute stroke were registered. Of these 364 (33\%) died in hospital, 211 (19\%) were discharged to residential or nursing homes, $92(9 \%)$ had non-disabling strokes or were discharged within a week of admission and 51 (5\%) lived outside the Nottingham Health District. Forty patients $(4 \%)$ were discharged before they could be allocated, $23(2 \%)$ were referred to a day hospital because of a need for relief care, 5 had been attending a hospital rehabilitation department before the stroke, 2 refused to give consent and 1 was discharged for terminal care only. Three patients had been included in the run-in phase of the study, and the remaining 327 patients were entered into the randomised trial.

The pre-randomisation characteristics of those allocated to the domiciliary and the hospital-based services are shown in table 1 . There were no important differences between the groups except that the Barthel scores at discharge were slightly higher in the hospitalbased group (Mann-Whitney, $p<0.05$ ). The latter difference was virtually confined to the General Medical stratum.

Patients in the Health Care of the Elderly stratum were 11 years older, on average, than those in the General Medical stratum and 17 
Table 1 Pre-randomisation characteristics of the study groups

\begin{tabular}{|c|c|c|c|c|c|c|c|c|}
\hline & \multicolumn{2}{|l|}{ Overall } & \multicolumn{2}{|l|}{$H C E$} & \multicolumn{2}{|l|}{$G M$} & \multicolumn{2}{|l|}{$S U$} \\
\hline & $D R S$ & $H R S$ & $D R S$ & HRS & $\overline{D R S}$ & $H R S$ & $D R S$ & HRS \\
\hline $\begin{array}{l}\text { Number } \\
\text { Age (mean) } \\
\text { Female } \\
\text { Living alone } \\
\text { Previous stroke } \\
\text { Previous immobility }\end{array}$ & $\begin{array}{l}162 \\
70 \\
77(48 \%) \\
58(36 \%) \\
40(25 \%) \\
35(22 \%)\end{array}$ & $\begin{array}{l}165 \\
70 \\
77(47 \%) \\
48(29 \%) \\
29(18 \%) \\
30(18 \%)\end{array}$ & $\begin{array}{l}79 \\
77 \\
41(52 \%) \\
34(43 \%) \\
26(33 \%) \\
29(37 \%)\end{array}$ & $\begin{array}{l}76 \\
77 \\
39(51 \%) \\
28(37 \%) \\
16(21 \%) \\
19(25 \%)\end{array}$ & $\begin{array}{l}58 \\
64 \\
21(36 \%) \\
18(31 \%) \\
10(17 \%) \\
5(9 \%)\end{array}$ & $\begin{array}{r}63 \\
67 \\
27(43 \%) \\
17(27 \%) \\
9(14 \%) \\
10(16 \%)\end{array}$ & $\begin{array}{l}25 \\
62 \\
15(60 \%) \\
6(24 \%) \\
4(16 \%) \\
1(4 \%)\end{array}$ & $\begin{array}{l}26 \\
58 \\
11(42 \%) \\
3(12 \%) \\
5(19 \%) \\
1(4 \%)\end{array}$ \\
\hline $\begin{array}{l}\text { Admission characteristics } \\
\text { Alert } \\
\text { Sensory deficit }\end{array}$ & $\begin{array}{r}144(89 \%) \\
28(17 \%)\end{array}$ & $\begin{array}{r}149(91 \%) \\
24(15 \%)\end{array}$ & $\begin{array}{l}71(90 \%) \\
12(15 \%)\end{array}$ & $\begin{array}{c}69(91 \%) \\
7(9 \%)\end{array}$ & $\begin{array}{r}53(91 \%) \\
9(16 \%)\end{array}$ & $\begin{array}{l}59(94 \%) \\
11(17 \%)\end{array}$ & $\begin{array}{r}20(80 \%) \\
7(28 \%)\end{array}$ & $\begin{array}{r}21(81 \%) \\
6(23 \%)\end{array}$ \\
\hline $\begin{array}{l}\text { Characteristics at study entry } \\
\text { Aphasia } \\
\text { Incontinence of urine } \\
\text { Abbreviated Mental Test } \\
\text { score-median (IQR) } \\
\text { Barthel score-median } \\
\text { (IQR) } \\
\text { Days in hospital } \\
\text { before randomisation } \\
\text {-median (IQR) }\end{array}$ & $\begin{array}{c}18(11 \%) \\
19(12 \%) \\
9.0 \\
(8-10) \\
16.0 \\
(14-17) \\
21 \\
(11-54)\end{array}$ & $\begin{array}{l}28(17 \%) \\
12(7 \%) \\
10 \\
(8-10) \\
17 \cdot 0 \\
(15-18) \\
18 \\
(10-51)\end{array}$ & $\begin{array}{c}8(10 \%) \\
14(18 \%) \\
9 \cdot 0 \\
(7-10) \\
16 \cdot 0 \\
(13-17) \\
21 \\
(12-54)\end{array}$ & $\begin{array}{l}13(17 \%) \\
8(11 \%) \\
9 \cdot 0 \\
(8-10) \\
17 \cdot 0 \\
(14-17) \\
26 \\
(11-52)\end{array}$ & $\begin{array}{l}5(9 \%) \\
2(3 \%) \\
10 \cdot 0 \\
(8-10) \\
16 \cdot 0 \star \\
(14-17) \\
13 \star \\
(9-21)\end{array}$ & $\begin{array}{l}5(8 \%) \\
3(5 \%) \\
10 \cdot 0 \\
(9-10) \\
17 \cdot 0 \\
(15-19) \\
10 \\
(7-15)\end{array}$ & $\begin{array}{c}5(20 \%) \\
3(12 \%) \\
10 \cdot 0 \\
(9-10) \\
16 \cdot 0 \\
(14-18) \\
103 \\
(72-132)\end{array}$ & $\begin{array}{l}10(39 \%) \\
1(4 \%) \\
10 \cdot 0 \\
(9-10) \\
17 \cdot 0 \\
(15-19) \\
89 \\
(64-118)\end{array}$ \\
\hline
\end{tabular}

^Mann-Whitney, $p<0.05$. HCE: Health Care of the Elderly stratum. GM: General Medical stratum. SU: Stroke Unit stratum. DRS: allocated to the domiciliary rehabilitation service. HRS: allocated to the hospital-based rehabilitation service. IQR: interquartile range.

years older than those discharged from the Stroke Unit, and a higher proportion of them lived alone (Chi-square, $\mathrm{p}<0.01$ ), had suffered a previous stroke (Chi-square, $p<$ $0.05)$ or had impaired mobility before the stroke (Chi-square, $p<0.01$ ). Stroke unit patients had a longer hospital stay than those in the Health Care of the Elderly or General Medical strata (Mann-Whitney, $\mathrm{p}<0.01$ ) and tended to have had more extensive strokes, as evidenced by the higher prevalence of aphasia (Chi-square, $p<0.01$ ).

REHABILITATION SERVICES

The provision of rehabilitation by the domiciliary and hospital-based services is shown in table 2. Patients allocated to the domiciliary service were more likely to receive some rehabilitation after discharge (Chi-square, $\mathrm{p}<$ 0.05 ), but amongst those who were treated the number of attendances in the first 6 months was higher in the routine care group (Mann-Whitney, p < 0.05). Overall, the numbers of treatment sessions provided by the domiciliary and hospital-based services were similar.

In the Health Care of the Elderly stratum "routine" care was provided at day hospitals for $28(78 \%)$ of the 36 patients who received treatment, while in the General Medical and Stroke Unit strata 53 patients allocated to the hospital-based service received treatment and this was carried out in an outpatient department in 51 cases.
Nine patients allocated to the domiciliary service actually received some treatment from the hospital-based services, but no patients allocated to the routine service received rehabilitation from the domiciliary team. In 5 cases a decision was made (after randomisation but before discharge) by a geriatrician that they should attend a day hospital. One patient who sustained a fractured neck of the femur during the study period was sent for outpatient therapy and one patient who suffered a further stroke was referred to a day hospital on discharge. In two cases, the domiciliary rehabilitation team referred patients to an occupational therapy department for workshop facilities.

\section{OUTCOME}

1 Death and place of residence

Table 3 shows overall outcome at 6 months in terms of survival and place of residence. There was a trend towards a higher death rate in the group allocated to the domiciliary service (relative risk $2 \cdot 3,95 \%$ confidence interval 1.0 to 5.5 ), and this trend was also seen in the Health Care of the Elderly stratum (relative risk $3.2,95 \%$ confidence interval 0.9 to $11 \cdot 0$ ). When "bad" outcome was defined as death, long term hospitalisation or a move into institutional care a similar trend was seen (relative risk 1.7, 95\% confidence interval 1.0 to 2.9 ), reaching significance in the Health Care of the Elderly stratum (relative risk $2 \cdot 4$, $95 \%$ confidence interval $1 \cdot 1$ to $5 \cdot 1$ ).

\begin{tabular}{|c|c|c|c|c|c|c|c|c|}
\hline & \multicolumn{2}{|l|}{ Overall } & \multicolumn{2}{|l|}{$H C E$} & \multicolumn{2}{|l|}{$G M$} & \multicolumn{2}{|l|}{$S U$} \\
\hline & $D R S$ & $H R S$ & $D R S$ & $H R S$ & $D R S$ & $H R S$ & $D R S$ & $H R S$ \\
\hline $\begin{array}{l}\text { Total number } \\
\text { Number receiving treatment } \\
\text { Attendances visits in } 6 \text { months median } \\
\text { Total number of attendances/visits in } 6 \text { months }\end{array}$ & $\begin{array}{l}162 \\
123(75 \%)^{\star} \\
7 \\
1615\end{array}$ & $\begin{array}{r}165 \\
89(54 \%) \\
16 \\
1626\end{array}$ & $\begin{array}{l}79 \\
59(75 \%)^{\star} \\
631\end{array}$ & $\begin{array}{l}76 \\
36(47 \%) \\
19 \\
726\end{array}$ & $\begin{array}{l}58 \\
39(67 \%) \\
6 \\
358\end{array}$ & $\begin{array}{l}63 \\
33(52 \%) \\
8 \\
446\end{array}$ & $\begin{array}{l}25 \\
25(100 \%)^{\star} \\
19 \\
626\end{array}$ & $\begin{array}{l}26 \\
\star \\
20(75 \%) \\
21 \\
454\end{array}$ \\
\hline
\end{tabular}

${ }^{\star} \mathrm{p}<0.05$. HCE: Health Care of the Elderly stratum. GM: General Medical stratum. SU: Stroke Unit stratum. DRS: allocated to the domiciliary rehabilitation service. HRS: allocated to the hospital-based rehabilitation service. 
Table 3 Overall outcome at 6 months

\begin{tabular}{|c|c|c|c|c|c|c|c|c|}
\hline & \multicolumn{2}{|l|}{ Overall } & \multicolumn{2}{|l|}{$H C E$} & \multicolumn{2}{|l|}{$G M$} & \multicolumn{2}{|l|}{$S U$} \\
\hline & $D R S$ & $H R S$ & $D R S$ & $H R S$ & $D R S$ & HRS & $D R S$ & $H R S$ \\
\hline $\begin{array}{l}\text { Home } \\
\text { In hospital } \\
\text { Residential/ nursing care } \\
\text { Dead } \\
\text { Bad outcome } \\
\text { "Expected" bad outcome } \\
\text { (see text for explanation) }\end{array}$ & $\begin{array}{c}134(82 \%) \\
3(2 \%) \\
9(6 \%) \\
16(10 \%) \\
28(18 \%) \\
27(17 \%)\end{array}$ & $\begin{array}{c}148(90 \%) \\
2(1 \%) \\
8(5 \%) \\
7(4 \%) \\
17(10 \%) \\
18(11 \%)\end{array}$ & $\begin{array}{r}59(75 \%) \\
1(1 \%) \\
9(11 \%) \\
10(13 \%) \\
20(25 \%) \\
22(28 \%)\end{array}$ & $\begin{array}{c}68(90 \%) \\
1(1 \%) \\
4(5 \%) \\
3(4 \%) \\
8(10 \%) \\
15(20 \%)\end{array}$ & $\begin{array}{c}51(88 \%) \\
2(3 \%) \\
0 \\
5(9 \%) \\
7(12 \%) \\
4(7 \%)\end{array}$ & $\begin{array}{c}58(92 \%) \\
0 \\
3(5 \%) \\
2(3 \%) \\
5(8 \%) \\
2(3 \%)\end{array}$ & $\begin{array}{c}24(96 \%) \\
0 \\
0 \\
1(4 \%) \\
1(4 \%) \\
1(4 \%)\end{array}$ & $\begin{array}{c}22(85 \%) \\
1(4 \%) \\
1(4 \%) \\
2(8 \%) \\
4(15 \%) \\
1(4 \%)\end{array}$ \\
\hline
\end{tabular}

$\star^{\star}$ Death or long term institutional nursing care. HCE: Health Care of the Elderly stratum. GM: General Medical stratum. SU: Stroke Unit stratum. DRS: allocated to the domiciliary rehabilitation service. HRS: allocated to the hospital-based SU: Stroke Unit straturition service.

At six months, 2 of the 5 patients who had been allocated to the domiciliary service but who were referred to a day hospital before hospital discharge had died, and one was discharged directly from hospital to a nursing home.

Using discriminant function analysis the following features were found to be independent predictors of bad outcome: increasing age, previous poor mobility, previous stroke, living alone, a sensory deficit on admission to hospital and a low mental test score at discharge. Table 1 shows that 5 of these $6 \mathrm{bad}$ prognostic factors were more prevalent in the group allocated to the domiciliary service. When adjustment was made for this imbalance and for the difference in the Barthel scores at discharge, the "expected" numbers of patients with bad outcome could be calculated (table 3). The "expected" and actual rates of bad outcome were very similar, indicating that much of the difference between the groups may be explained by allocation bias.

\section{Disability}

Extended $\mathrm{ADL}$ questionnaires were available for analysis in 299 patients at three months and 303 patients at six months after randomisation. Of the 18 non-responders from the domiciliary group at six months, 15 had died, one refused to complete the questionnaire, one had moved to a distant nursing home and did not reply and one could not be contacted. All 6 non-responders from the routine hospital service group at six months had died. One patient allocated to each service died between completing the postal questionnaire and the assessment at six months. The Extended ADL scores for those who responded at three and six months are shown in table 4. There was no difference in the total Extended ADL scores between the groups (median difference $0.0,95 \%$ confidence interval -1 to 1 , Mann-Whitney, $p>$ 0.05 ). The only significant differences (in favour of the domiciliary group) were in household (median difference 2.0, 95\%

Table 4 Barthel and Extended ADL scores

\begin{tabular}{|c|c|c|c|c|c|c|c|c|}
\hline & \multicolumn{2}{|l|}{ Overall } & \multicolumn{2}{|l|}{$H C E$} & \multicolumn{2}{|l|}{$G M$} & \multicolumn{2}{|l|}{$S U$} \\
\hline & $D R S$ & HRS & $D R S$ & $H R S$ & $D R S$ & HRS & $D R S$ & $H R S$ \\
\hline \multicolumn{9}{|c|}{$\begin{array}{l}\text { Extended ADL-total } \\
3 \text { months }\end{array}$} \\
\hline $\begin{array}{l}\text { median } \\
\text { (IQR) }\end{array}$ & $\begin{array}{l}8 \cdot 0 \\
(4-13)\end{array}$ & $\begin{array}{l}8 \cdot 5 \\
(4-13)\end{array}$ & $\begin{array}{c}6 \cdot 0 \\
(2-10)\end{array}$ & $\begin{array}{c}8 \cdot 0 \\
(2-11)\end{array}$ & $\begin{array}{l}11 \cdot 0 \\
(6-16)\end{array}$ & $\begin{array}{l}11 \cdot 0 \\
(6-16)\end{array}$ & $\begin{array}{l}7 \cdot 0 \\
(5-11 \cdot 5)\end{array}$ & $\begin{array}{c}6 \cdot 5 \\
(5-9)\end{array}$ \\
\hline $\begin{array}{c}6 \text { months } \\
\text { median } \\
\text { (IQR) }\end{array}$ & $\begin{array}{c}8 \cdot 5 \\
(4-14)\end{array}$ & $\begin{array}{c}8 \cdot 0 \\
(4-14)\end{array}$ & $\begin{array}{l}6 \cdot 0 \\
(3-10)\end{array}$ & $\begin{array}{c}8 \cdot 0 \\
(4-12)\end{array}$ & $\begin{array}{l}12 \cdot 5 \\
(7-17)\end{array}$ & $\begin{array}{l}12 \cdot 0 \\
(6-17 \cdot 5)\end{array}$ & $\begin{array}{l}9 \cdot 5 \\
(5-12)\end{array}$ & $\begin{array}{l}6 \cdot 0 \\
(3 \cdot 5-10 \cdot 5)\end{array}$ \\
\hline \multicolumn{9}{|c|}{$\begin{array}{l}\text { Extended ADL-mobility } \\
3 \text { months }\end{array}$} \\
\hline median & $\begin{array}{c}2 \cdot 0 \\
(0-4)\end{array}$ & $\begin{array}{c}2 \cdot 0 \\
(0-5)\end{array}$ & $\begin{array}{c}1 \cdot 0 \\
(0-3)\end{array}$ & $\begin{array}{c}1 \cdot 0 \\
(0-4)\end{array}$ & $\begin{array}{c}4 \cdot 0 \\
(1-6)\end{array}$ & $\begin{array}{c}4 \cdot 0 \\
(1-5)\end{array}$ & $\begin{array}{c}1 \cdot 0 \\
(0-3)\end{array}$ & $\begin{array}{c}2 \cdot 0 \\
(1-4)\end{array}$ \\
\hline $\begin{array}{l}6 \text { months } \\
\text { median } \\
\text { (IQR) }\end{array}$ & $\begin{array}{c}2 \cdot 0 \\
(0-5)\end{array}$ & $\begin{array}{c}2 \cdot 0 \\
(0-5)\end{array}$ & $\begin{array}{c}1 \cdot 0 \\
(0-3)\end{array}$ & $\begin{array}{c}1 \cdot 0 \\
(0-4)\end{array}$ & $\begin{array}{c}4 \cdot 0 \\
(2-6)\end{array}$ & $\begin{array}{l}4 \cdot 0 \\
(1-5 \cdot 5)\end{array}$ & $\begin{array}{l}1 \cdot 5 \\
(0-4)\end{array}$ & $\begin{array}{l}1 \cdot 0 \\
(0-4 \cdot 5)\end{array}$ \\
\hline \multicolumn{9}{|c|}{$\begin{array}{l}\text { Extended ADL-household } \\
3 \text { months }\end{array}$} \\
\hline $\begin{array}{l}\text { median } \\
\text { (IQR) } \\
6 \text { months }\end{array}$ & $\begin{array}{c}4 \cdot 0 \\
(2-7)\end{array}$ & $\begin{array}{c}4 \cdot 0 \\
(2-6)\end{array}$ & $\begin{array}{c}3 \cdot 0 \\
(1-6)\end{array}$ & $\begin{array}{c}4 \cdot 0 \\
(1-6)\end{array}$ & $\begin{array}{c}4 \cdot 5 \\
(3-8)\end{array}$ & $\begin{array}{c}5 \cdot 0 \\
(2-8)\end{array}$ & $\begin{array}{l}4 \cdot 0 \\
(3-6 \cdot 5)\end{array}$ & $\begin{array}{c}3 \cdot 0 \\
(2-4)\end{array}$ \\
\hline median & $\begin{array}{c}4 \cdot 0 \\
(2-7)\end{array}$ & $\begin{array}{c}4 \cdot 0 \\
(2-7)\end{array}$ & $\begin{array}{c}3 \cdot 0 \\
(1-6)\end{array}$ & $\begin{array}{c}4 \cdot 0 \\
(2-6)\end{array}$ & $\begin{array}{c}6 \cdot 0 \\
(3-7)\end{array}$ & $\begin{array}{l}6 \cdot 0 \\
(2-8 \cdot 5)\end{array}$ & $\underset{(3-7)}{5 \cdot 0}$ * & $\begin{array}{l}3.0 \\
(0.5-4 \cdot 5)\end{array}$ \\
\hline \multicolumn{9}{|c|}{$\begin{array}{l}\text { Extended ADL-leisure } \\
3 \text { months }\end{array}$} \\
\hline $\begin{array}{l}\text { median } \\
\text { (IQR) }\end{array}$ & $\begin{array}{c}2 \cdot 0 \\
(1-3)\end{array}$ & $\begin{array}{c}2 \cdot 0 \\
(1-3)\end{array}$ & $\begin{array}{c}2 \cdot 0 \\
(1-3)\end{array}$ & $\begin{array}{c}2 \cdot 0 \\
(1-3)\end{array}$ & $\begin{array}{c}2 \cdot 5 \\
(1-4)\end{array}$ & $\begin{array}{c}2 \cdot 0 \\
(2-4)\end{array}$ & $\begin{array}{c}2 \cdot 0 \\
(1-3)\end{array}$ & $\begin{array}{c}1.5 \\
(1-3)\end{array}$ \\
\hline $\begin{array}{l}\text { median } \\
\text { (IQR) }\end{array}$ & $\begin{array}{c}2 \cdot 0 \\
(2-3)\end{array}$ & $\begin{array}{c}2 \cdot 0 \\
(1-3)\end{array}$ & $\begin{array}{c}2 \cdot 0 \\
(1-3)\end{array}$ & $\begin{array}{c}2 \cdot 0 \\
(1-3)\end{array}$ & $\begin{array}{c}3 \cdot 0 \\
(2-4)\end{array}$ & $\begin{array}{c}2 \cdot 0 \\
(1-4)\end{array}$ & $\underset{(2-3)}{2 \cdot 5}$ * & $\begin{array}{l}2 \cdot 0 \\
(1-2 \cdot 5)\end{array}$ \\
\hline \multicolumn{9}{|c|}{$\begin{array}{l}\text { BARTHEL-ADL } \\
\text { randomisation }\end{array}$} \\
\hline $\begin{array}{l}\text { median } \\
\text { (IQR) }\end{array}$ & $\begin{array}{l}16 \cdot 0 \\
(14-17)\end{array}$ & $\begin{array}{l}17 \cdot 0 \\
(15-18)\end{array}$ & $\begin{array}{l}16 \cdot 0 \\
(13-17)\end{array}$ & $\begin{array}{l}17 \cdot 0 \\
(14-17)\end{array}$ & $\begin{array}{l}16 \cdot 0 \\
(14-17)\end{array}$ & $\begin{array}{l}17 \cdot 0 \\
(15-19)\end{array}$ & $\begin{array}{l}16 \cdot 0 \\
(14-18)\end{array}$ & $\begin{array}{l}17 \cdot 0 \\
(15-19)\end{array}$ \\
\hline median & $\begin{array}{l}17 \cdot 0 \\
(14-19)\end{array}$ & $\begin{array}{l}18 \cdot 0 \\
(15-20)\end{array}$ & $\begin{array}{l}17 \cdot 0 \\
(12-18)\end{array}$ & $\begin{array}{l}17 \cdot 0 \\
(14-19)\end{array}$ & $\begin{array}{l}19 \cdot 0 \\
(16-20)\end{array}$ & $\begin{array}{l}19 \cdot 0 \\
(17-20)\end{array}$ & $\begin{array}{l}18 \cdot 0 \\
(15-19)\end{array}$ & $\begin{array}{l}16 \cdot 0 \\
(15-18)\end{array}$ \\
\hline
\end{tabular}

${ }^{\star} \mathrm{p}<0.05$. HCE: Health Care of the Elderly stratum. GM: General Medical stratum. SU: Stroke Unit stratum. DRS: allocated to the domiciliary rehabilitation service. HRS: allocated to the hospital-based rehabilitation service. IQR: interquartile range. 
Table 5 Nottingham Health Profile scores at six months. (Possible scores in each domain range between 0 and 100, higher scores denote worse perceived health status)

\begin{tabular}{|c|c|c|c|c|c|c|c|c|}
\hline & \multicolumn{2}{|l|}{ Overall } & \multicolumn{2}{|l|}{$H C E$} & \multicolumn{2}{|l|}{$G M$} & \multicolumn{2}{|l|}{$S U$} \\
\hline & $D R S$ & HRS & $D R S$ & $H R S$ & $D R S$ & HRS & $D R S$ & HRS \\
\hline \multicolumn{9}{|l|}{ ENERGY } \\
\hline $\begin{array}{l}\text { median } \\
\text { (IQR) } \\
\text { EMOTIONS }\end{array}$ & $\begin{array}{l}24 \\
(0-63)\end{array}$ & $\begin{array}{l}24 \\
(0-61)\end{array}$ & $\begin{array}{l}37 \\
(0-100)\end{array}$ & $\begin{array}{l}38 \\
(0-63)\end{array}$ & $\begin{array}{l}37 \\
(12-63)\end{array}$ & $\begin{array}{l}24 \\
(0-61)\end{array}$ & $\stackrel{0}{(0-24)}$ & $\stackrel{0}{0}+(0-61)$ \\
\hline $\begin{array}{l}\text { median } \\
\text { (IQR) }\end{array}$ & $\begin{array}{l}10 \\
(0-41)\end{array}$ & $\begin{array}{l}14 \\
(0-44)\end{array}$ & $\begin{array}{l}10 \\
(0-46)\end{array}$ & $\begin{array}{l}16 \\
(0-46)\end{array}$ & $\begin{array}{l}23 \\
(0-44)\end{array}$ & $\begin{array}{l}10 \\
(0-44)\end{array}$ & $\begin{array}{l}0 \\
(0-17)\end{array}$ & $\begin{array}{l}10 \\
(0-42)\end{array}$ \\
\hline median & $\begin{array}{l}16 \\
(0-50)\end{array}$ & $\begin{array}{l}13 \\
(0-35)\end{array}$ & $\begin{array}{l}16 \\
(0-50)\end{array}$ & $\begin{array}{l}13 \\
(0-38)\end{array}$ & $\begin{array}{l}22 \\
(0-67)\end{array}$ & $\begin{array}{l}13 \\
(0-35)\end{array}$ & $\begin{array}{l}13 \\
(0-35)\end{array}$ & $\begin{array}{l}13 \\
(0-22)\end{array}$ \\
\hline $\begin{array}{l}\text { ISOLATION } \\
\text { median } \\
\text { (IQR) }\end{array}$ & $\begin{array}{l}19 \\
(0-23)\end{array}$ & $\begin{array}{l}20 \\
(0-42)\end{array}$ & $\begin{array}{l}22 \\
(0-45)\end{array}$ & $\begin{array}{l}19 \\
(0-42)\end{array}$ & $\begin{array}{l}16 \\
(0-23)\end{array}$ & $\begin{array}{l}22 \\
(0-41)\end{array}$ & $\stackrel{0}{(0-23)}$ & $\begin{array}{l}22 \\
(0-55)\end{array}$ \\
\hline $\begin{array}{l}\text { PAIN } \\
\text { median } \\
\text { (IQR) } \\
\text { PHYSICAL MOBILITY }\end{array}$ & $\begin{array}{l}11 \\
(0-30)\end{array}$ & $\begin{array}{l}16 \\
(0-23)\end{array}$ & $\begin{array}{l}17 \\
(0-30)\end{array}$ & $\begin{array}{l}13 \\
(0-21)\end{array}$ & $\begin{array}{l}0 \\
(0-31)\end{array}$ & $\begin{array}{l}0 \\
(0-23)\end{array}$ & $\stackrel{1}{(0-15)}$ & $\begin{array}{l}0 \\
(0-28)\end{array}$ \\
\hline $\begin{array}{l}\text { median } \\
\text { (IQR) }\end{array}$ & $\begin{array}{l}36 \\
(13-58)\end{array}$ & $\begin{array}{l}33 \\
(11-55)\end{array}$ & $\begin{array}{l}46 \\
(17-67)\end{array}$ & $\begin{array}{l}35 \\
(21-66)\end{array}$ & $\begin{array}{l}33 \\
(11-55)\end{array}$ & $\begin{array}{l}23 \\
(11-47)\end{array}$ & $\begin{array}{l}29 \\
(10-59)\end{array}$ & $\begin{array}{l}33 \\
(11-51)\end{array}$ \\
\hline
\end{tabular}

HCE: Health Care of the Elderly stratum. GM: General Medical stratum. SU: Stroke Unit stratum. DRS: allocated to the domiciliary rehabilitation service. HRS: allocated to the hospital-based rehabilitation service. IQR: interquartile range.

confidence interval 0 to 3, Mann-Whitney, $\mathrm{p}<0.05$ ) and leisure scores (median difference $1 \cdot 0,95$ confidence interval 0 to 2 , Mann-Whitney, $\mathrm{p}<0.05$ ) at six months for patients discharged from the Stroke Unit. In this stratum the improvement in total Extended ADL scores between three and six months after discharge was also greater in the group treated at home (median difference $2 \cdot 0,95 \%$ confidence interval 1 to 4 , MannWhitney, $\mathrm{p}<0.01$ ).

Multivariate regression analysis revealed six independent predictors of the total Extended ADL score: Barthel and mental test score at discharge, decreasing age, previous mobility, shorter hospital stay and living alone. Adjusting for the base-line differences in these risk factors using covariance analysis, confirmed that there was no significant effect of treatment allocation, either overall $(p=$ 0.29 ), or within the Health Care of the Elderly or General Medical strata separately, but in the patients discharged from the Stroke Unit allocation to the domiciliary team was associated with significantly better Extended ADL scores at six months $(p=0.02)$.

Barthel scores were obtained for 300 patients at 6 months and the results are shown in table 4. Reasons for missed assessments were as for the Extended ADL. There were no differences between final Barthel scores of the groups overall (median differ- ence $0 \cdot 0,95 \%$ confidence interval -1 to 0 , Mann-Whitney, p>0.05), but Stroke Unit patients treated by the domiciliary team showed significantly greater improvements between randomisation and six months (median difference $1 \cdot 0,95 \%$ confidence interval 0 to 3, Mann-Whitney, $\mathrm{p}<0.01$ ).

\section{Perceived health}

The Nottingham Health Profile (NHP) was completed by 271 patients. Of the 56 nonresponders, 23 died, 13 were dysphasic, 18 were deaf and unable to read the test statements and 2 were not contacted. The results are shown in table 5 . No significant differences in NHP scores were found between the groups, either overall or within individual strata.

\section{Carer social engagement and life satisfaction}

The Brief Assessment of Social Engagement and Life Satisfaction Index were completed by 180 and 178 carers respectively and the results are shown in table 6 . There were no differences between the scores of the carers of patients allocated to the domiciliary or the hospital based service.

\section{Discussion}

This is one of the largest randomised trials specifically concerned with stroke rehabilitation yet reported in the UK. A high

Table 6 Brief Assessment of Social Engagement (BASE) and Life Satisfaction Index (N-LSIZ) scores in carers. (BASE scores vary between 0 and 20, higher scores denoting greater social engagement. N-LSIZ scores vary between 0 and 26, higher scores denoting greater life satisfaction)

\begin{tabular}{|c|c|c|c|c|c|c|c|c|}
\hline & \multicolumn{2}{|l|}{ Overall } & \multicolumn{2}{|l|}{$H C E$} & \multicolumn{2}{|l|}{$G M$} & \multicolumn{2}{|l|}{$S U$} \\
\hline & $D R S$ & $H R S$ & $\overline{D R S}$ & $H R S$ & $\overline{D R S}$ & $H R S$ & $\overline{D R S}$ & $H R S$ \\
\hline $\begin{array}{l}\text { BASE } \\
\text { n } \\
\text { median } \\
\text { IQR }\end{array}$ & $\begin{array}{l}77 \\
12 \cdot 0 \\
(11-15)\end{array}$ & $\begin{array}{l}103 \\
13 \cdot 0 \\
(11-15)\end{array}$ & $\begin{array}{l}35 \\
12 \cdot 0 \\
(10-13)\end{array}$ & $\begin{array}{l}39 \\
13 \cdot 0 \\
(11-15)\end{array}$ & $\begin{array}{l}28 \\
12 \cdot 5 \\
(11-14)\end{array}$ & $\begin{array}{l}45 \\
13 \cdot 0 \\
(11-17)\end{array}$ & $\begin{array}{l}14 \\
14 \cdot 0 \\
(11-16)\end{array}$ & $\begin{array}{l}19 \\
14 \cdot 0 \\
(12-16)\end{array}$ \\
\hline $\begin{array}{l}\text { n-LSIL } \\
\text { median } \\
\text { IQR }\end{array}$ & $\begin{array}{l}76 \\
17 \cdot 0 \\
(12-20)\end{array}$ & $\begin{array}{l}102 \\
18 \cdot 0 \\
(12-22)\end{array}$ & $\begin{array}{l}34 \\
15 \cdot 0 \\
(10-19)\end{array}$ & $\begin{array}{l}39 \\
18 \cdot 0 \\
(11-22)\end{array}$ & $\begin{array}{l}28 \\
18 \cdot 0 \\
(12-21)\end{array}$ & $\begin{array}{l}44 \\
18 \cdot 0 \\
(14-22)\end{array}$ & $\begin{array}{l}14 \\
17 \cdot 5 \\
(14-22)\end{array}$ & $\begin{array}{l}19 \\
18 \cdot 0 \\
(12-24)\end{array}$ \\
\hline
\end{tabular}

HCE: Health Care of the Elderly stratum. GM: General Medical stratum. SU: Stroke Unit stratum. DRS: allocated to the domiciliary rehabilitation service. HRS: allocated to the hospital-based rehabilitation service. IQR: interquartile range. 
proportion of the stroke patients discharged from all the medical and geriatric wards in Nottingham was recruited, so the study sample may well be representative of stroke patients discharged from other British hospitals.

In everyday clinical practice, domiciliary and hospital-based rehabilitation services are likely to differ in many respects, such as the proportion and type of patients selected for further treatment, the pattern and intensity of therapy, and the range of other facilities available. To identify the independent effects of these separate factors on outcome would require an "explanatory" trial, ${ }^{23}$ where each factor is carefully controlled, thus imposing artificial conditions on the rehabilitation services. We chose to adopt the alternative "pragmatic" approach, ${ }^{23}$ comparing the two services as total "packages" operating under realistic conditions. In effect, we performed 3 separate randomised trials since the characteristics of the patients and the services provided differed so much between the 3 strata. Thus we have chosen to analyse and interpret the results separately in the 3 groups, while accepting that this reduces the statistical confidence of the conclusions.

Considerably more patients received treatment under the domiciliary service than the usual hospital-based service, although the total number of treatment sessions was similar. Overall outcomes were similar in terms of the functional ability and perceived health status of the patients, and the degree of social engagement and life satisfaction of their carers. Although domiciliary rehabilitation for stroke is not always available, for example in Nottingham before and after this trial, it is a viable proposition and is applicable to a high proportion of stroke patients.

Within the wide range of patients in the DOMINO study, there was a sub-group of frail elderly patients who appeared to fare better with day hospital rather than domiciliary care. This benefit of day hospital care (if it is not spurious due to the small group size and baseline difference between the groups) may relate to the multidisciplinary nature of day hospital care given to these selected patients, or it may be due to the greater intensity of treatment given to them by the day hospital service. These results conflict with the results of the Bradford community stroke trial ${ }^{10}$ in which 124 stroke patients were randomly allocated at discharge to receive further therapy from a community physiotherapist or in a day hospital. Patients in the Bradford study who were treated at home showed a greater improvement in ADL ability than the day hospital group, and this advantage was maintained after six months of follow up. The discrepancy may be explained by differences in the characteristics of the patients treated in the two studies and in the services provided. The Bradford patients were seven years younger, on average, than patients in the Health Care of the Elderly stratum of the DOMINO study, so they were probably less frail. Moreover, all patients in
Bradford received treatment compared with $75 \%$ of the Nottingham Health Care of the Elderly patients, 5 therapists were available in Bradford as opposed to 2 in Nottingham and treatment was continued for a minimum of eight weeks in the Bradford trial whereas no lower limit was imposed in our study.

In contrast to the frail elderly, the smaller group of Stroke Unit patients seemed to do better with domiciliary rehabilitation. It is in these younger patients with severe strokes yet with little previous disability that we believe rehabilitation has the greatest potential. Furthermore, as might be expected, the impact of domiciliary rehabilitation appeared greatest in terms of household and leisure abilities, so we believe that this is a clinically important finding. With an average of less than one visit per week, the domiciliary therapists were able to prevent the functional deterioration commonly seen after discharge in the Edinburgh Stroke Unit trial ${ }^{3}$ and indeed achieved similar improvements to those seen in the intensive therapy group of the Northwick Park outpatient rehabilitation trial. ${ }^{4}$

As with other rehabilitation studies, it is difficult to be certain whether the results of the DOMINO study can be generalised to rehabilitation services provided elsewhere. Similar trials should be undertaken in different settings before general conclusions can be reliably drawn: indeed, all rehabilitation centres should be encouraged to subject their practice to scientific scrutiny.

Overall we conclude that domiciliary rehabilitation in Nottingham proved a practical and effective alternative to outpatient department therapy for younger patients with severe strokes, and an efficient way to cater for the needs of patients with milder strokes after discharge from hospital. Great care should be taken in selecting frail elderly patients for domiciliary rehabilitation, however, since some of them may require medical supervision and maintenance care in a geriatric day hospital. Further studies are required to clarify the situation in this numerically large and important group.

We are grateful for financial support from the Chest, Heart and Stroke Association, the Nottingham Fights Stroke Association, the Medical Research Council (grant number G8813670) and the Rehabilitation and Medical Research Trust. We would like to thank the rehabilitation, medical and nursing staff in Nottingham who cooperated with this study.
Thanks to Mrs L Creighton of the Cripps Computing Centre, University of Nottingham for help with computing.

1 Garraway WM, Akhtar AJ, Prescott RJ, Hockey L. Management of acute stroke in the elderly: preliminary Management of acute stroke in the elderly: prelim
results of a controlled trial. $B M F$ 1980;280:1040-3.

2 Indredavik B, Bakke F, Solberg R, Rokseth R, Haaheim LL, Holme I. Benefit of a stroke unit: a randomized co, Holme I. Benefit of a stroke unit:

3 Garraway WM, Akhtar AJ, Hockey L, Prescott RI. Management of acute stroke in the elderly: follow-up of a controlled trial. BMF 1980;281:827-9.

4 Smith DL, Goldenberg E, Ashburn A, Kinsella G, Sheikh $\mathrm{K}$, et al. Remedial therapy after stroke: a randomised controlled trial. $B M \mathcal{F}$ 1981;282:517-20.

5 Sheikh K, Meade TW, Brennan PJ, Goldenberg E, Smith DS. Intensive rehabilitation after stroke: service implications. Community Med 1981;3:210-16.

6 Ebrahim S, Nouri F. Caring for stroke patients at home. Int Rehabil Med 1987;8:171-3. 
7 Ebrahim SB, Barer DH, Nouri F. An audit of follow up services for stroke patients after discharge from hospital. Int Disabil Studies 1987;9:103-5.

8 Stokoe D, Zuccollo G. Travel sickness in patients attending a geriatric day hospital. Age Ageing 1985;14: 308-11.

9 Wade DT, Langton Hewer R, Skilbeck CE, Bainton D, Burns-Cox C. Controlled trial of a home care service for acute stroke patients. Lancet $1985 ; \mathrm{i}: 323-6$.

10 Young JB, Forster A. The Bradford community stroke trial: results at six months. BMF 1992;304:1085-9.

11 Aho K, Harmsen P, Hatano S, Marquardson J, Smirnov VE, Strasser T. Cerebrovascular disease in the community: results of a WHO collaborative study. Bull WHO 1980;58:113-30.

12 Mahoney FI, Barthel DW. Functional evaluation: the Barthel Index. Maryland State Med $₹$ 1965;14:61-5.

13 Collin C, Wade DT, Davies S, Horne V. The Barthel ADL index: a reliability study. Int Disabil Stud 1988; 10:61-3.

14 Hodkinson HM. Evaluation of a mental test score for assessment of mental impairment in the elderly. Age Ageing 1972;1:233-8.

15 Gladman JRF, Lomas S, Lincoln NB. Provision of physiotherapy and occupational therapy to outpatien departments and day hospitals for stroke patients in departments and day hospitals for stroke pat

16 Nouri F, Lincoln NB. An extended activities of daily living scale for stroke patients. Clinical Rehabilitation 1987; $1: 301-5$

17 Lincoln NB, Gladman JRF. The Extended Activities of Daily Living Scale: a further validation. Disability and Rehabilitation 1992;14:41-3.

18 Hunt SM, McEwan J, McKenna SP. Measuring health status. London: Croom Helm, 1986.

19 Morgan K, Dallosso HM, Ebrahim SBJ. A brief selfreport scale for assessing personal engagement in the elderly: reliability and validity. In: A Butler, ed. Ageing. London: Croom Helm, 1985.

20 Morgan K, Dallosso HM, Arie T, Byrne EJ, Jones R Waite J. Mental health and well-being among the old Waite $\mathrm{J}$. Mental health and well-being among the old
and very old living at home. $\mathrm{Br} \mathcal{F}$ Psychiatry 1987;150: and ve

21 Neugarten BL, Havighurst RJ, Tobin SS. The measurement of life satisfaction. $\mathcal{F}$ Gerontol 1961;16:134-43.

22 Kirkwood BR. Essentials of medical statistics. Oxford: Blackwell, 1988:191-200.

23 Schwartz D, Lellouche J. Explanatory and pragmatic attitudes in therapeutic trials. $f$ Chronic Disease $1967 ; 20$ 637-48. 